\title{
Research on Fault Diagnosis Optimization of Intelligent Acquisition Terminal
}

\author{
Xinran Liu ${ }^{1}$, Liyan Kang ${ }^{2}$, Ying Shang ${ }^{3}$, Lina Sun ${ }^{4}$, Zhongcheng Li $^{5}$, Qiutong Wu ${ }^{6}$, Muxin Zhang ${ }^{7}$, Hecheng Wang ${ }^{8}$ \\ ${ }^{1}$ State Grid Liaoning Electric Power Co., Ltd. Institute of Electric Power Science, Shenyang, 110003, China \\ ${ }^{2}$ State Grid Liaoning Electric Power Co., Ltd. Institute of Electric Power Science, Shenyang, 110003, China \\ ${ }^{3}$ State Grid Liaoning Electric Power Co., Ltd. Institute of Electric Power Science, Shenyang, 110003, China \\ ${ }^{4}$ State Grid Liaoning Electric Power Co., Ltd. Institute of Electric Power Science, Shenyang, 110003, China \\ ${ }^{5}$ State Grid Liaoning Electric Power Co., Ltd. Institute of Electric Power Science, Shenyang, 110003, China \\ ${ }^{6}$ State Grid Liaoning Electric Power Co., Ltd. Institute of Electric Power Science, Shenyang, 110003, China \\ ${ }^{7}$ State Grid Liaoning Electric Power Co., Ltd. Institute of Electric Power Science, Shenyang, 110003, China \\ ${ }^{8}$ State Grid Shenyang Power Supply Co., Ltd. Shenyang, 110000, China
}

\begin{abstract}
Intelligent acquisition terminal is an important medium for power users to collect electric energy data and load data. It realizes centralized collection of electricity information through local and remote communication technology. With the continuous development of communication technology, the acquisition terminal is becoming more and more intelligent and its functions are becoming more and more complex. The content of field equipment fault diagnosis analysis technology is getting higher and higher. This paper combines with the current intelligent acquisition terminal fault diagnosis mode to optimize the research, from the technical and management aspects, makes an analysis of the causes of acquisition anomalies and the lack of means, and puts forward reasonable efficiency improvement suggestions.
\end{abstract}

\section{Introduction}

As an important part of smart grid, electric power marketing measurement is an important link of electric power production, management and safe operation of power grid. Its technology and management level directly affects the fairness, fairness, accuracy and reliability of trade settlement, and relates to the vital interests of the vast number of power users, as well as the high-quality service level of power enterprises. The State Grid Co., Ltd. has vigorously promoted the application of smart watt-hour meters, intelligent acquisition terminals and telecommunication information acquisition system, and achieved the construction objectives of "automatic verification, automatic acquisition, online monitoring and intelligent diagnosis" of metrology.

Acquisition terminal is an important medium for power users to collect electric energy data and load data. It is a device for collecting electric energy information at various electric power information collection points. It can realize data acquisition, data management, data bidirectional transmission and forwarding or executing control commands. In addition to the above basic functions, intelligent acquisition terminal also has the ability of perception expansion and powerful edge. Edge computing power. The function of intelligent acquisition terminal is becoming more and more complex, the content of fault diagnosis and analysis technology of field equipment is getting higher and higher, and the technical level requirement of grass-roots operation and maintenance personnel is gradually increasing. The fault intelligent diagnosis model developed by fully applying marketing big data analysis can provide technical guidance for field operation and maintenance personnel, reduce the technical threshold of operation and maintenance personnel, play the purpose of assistant decision-making and reduce the fault. Point range, give reasonable suggestions to eliminate deficiencies, greatly improve the quality and efficiency of work.[1]

\section{Current Situation of Fault Diagnosis Mode for Intelligent Acquisition Terminal}

\subsection{Development Direction of Electricity Information Collection Specialty}

With the advancement of smart grid construction, the application scope of data collected by electricity information is expanding, the business support ability is improving, and the attention of the system is also increasing. At present, 69 data items have been collected in full. The collection frequency of some data items has been raised from daily freezing to hourly level. The step-by-step increase in the amount of data collected is a test for the main channel of acquisition station, including 
acquisition equipment. It is also a new step and a new leap in acquisition work.

With the gradual completion of the acquisition coverage work, the focus of acquisition work is gradually shifting from system construction to system operation and maintenance. In this new direction of work and the increasingly fierce competition in the power market, power marketing personnel urgently need to rely on information technology to provide accurate services for users to ensure the stable operation of equipment. It has become the target of intelligent acquisition and maintenance of acquisition system to detect all kinds of abnormalities in time through massive data, to find hidden troubles in advance, to complete on-site disposal as early as possible, and to improve the quality and efficiency of operation and maintenance.

\subsection{Fault Diagnosis Mode of Intelligent Acquisition Terminal at Present}

At present, the principle of acquisition absence is mainly divided into master station analysis, acquisition terminal analysis and communication analysis. Its mode and existing problems are shown in the table below.

Table 1. Fault Diagnosis Methods and Existing Problems of Data Acquisition at the Present Stage

\begin{tabular}{ll}
\hline $\begin{array}{c}\text { Process of } \\
\text { eliminating } \\
\text { defects }\end{array}$ & \multicolumn{1}{c}{$\begin{array}{c}\text { Collection and Fault Diagnosis at the } \\
\text { Present Stage }\end{array}$} \\
\hline & $\begin{array}{l}\text { When diagnosing and analyzing } \\
\text { acquisition faults, it is necessary to } \\
\text { judge whether the relevant } \\
\text { communication of acquisition terminal } \\
\text { 1 Master station } \\
\text { analysis }\end{array}$ \\
$\begin{array}{l}\text { is normal and whether the contents of } \\
\text { archives and parameters are correct by } \\
\text { means of data call, heartbeat check and } \\
\text { archives from the main station side.[2] }\end{array}$ \\
$\begin{array}{l}\text { For those who can not find out the } \\
\text { cause of the problem from the main } \\
\text { terminal and } \\
\text { communication } \\
\text { analysis }\end{array}$ & $\begin{array}{l}\text { station, they can go to the scene to } \\
\text { check the acquisition terminal, check } \\
\text { the terminal parameter settings, RS485 } \\
\text { communication, etc. }\end{array}$ \\
\hline
\end{tabular}

\begin{tabular}{ll}
\hline & $\begin{array}{l}\text { Distribution of fault diagnosis work } \\
\text { orders requires manual arrangement }\end{array}$ \\
and issuance by supervisory managers. \\
means & The procedure of dispatching work \\
& orders is complex and it is not easy to \\
track the feedback results of \\
dispatching work orders.
\end{tabular}

The discovery and analysis of anomalies are manually derived and analyzed by the operator of the main station. Basically, they are only aimed at the areas where the collection rate has decreased or the users have not been copied.

Completely depending on the experience of field operation and maintenance personnel for infrared replication or setup, the elimination of field faults from the appearance, there may be a lot of misdiagnosis, misoperation and repetitive work.

On-site fault diagnosis is mostly done by printing paper documents manually. Operating and maintenance personnel fill in and eliminate the defects on-site according to their own experience, and then transfer them to monitoring personnel. It is difficult to form closed-loop management because of the lack of results tracking ability of supervisory managers.

inefficient operation and maintenance work and may not fundamentally solve the problem.

Second, from the management point of view: the discovery and analysis of anomalies are manually derived and analyzed by the mainstay operators, and the coverage is not complete. Including processing means, time and processing results are not easy to track, monitoring managers lack effective management means and ways for the entire process, lack of follow-up ability, and it is difficult to form closed-loop management. The evaluation of operation and maintenance personnel and equipment manufacturers is mainly based on the single and rough assessment of the success rate of acquisition, which can not effectively carry out detailed analysis of operation and maintenance indicators. 


\section{$3 \quad$ Fault Diagnosis Model of Intelligent Acquisition Terminal Based on Large Data Analysis}

Based on the above problems, the traditional fault treatment mode can not meet the growing demand for operation and maintenance. It is necessary to innovate the diagnosis mode to ensure the safe, stable and reliable operation of field equipment. The fault diagnosis model of intelligent acquisition terminal based on large data analysis collects power consumption information and field fault information comprehensively, fully investigates the demand of field fault treatment technology, and studies the diagnosis model by using large data analysis and artificial intelligence technology, so as to solve the problems of precise diagnosis of acquisition fault, qualitative analysis of equipment fault and guidance of field fault treatment process.

\subsection{Establishment of Fault Diagnosis Model for Intelligent Acquisition Terminal}

Through collecting and sorting out fault types, collecting on-line monitoring of measurement anomalies, collecting problems in fault site processing and optimizing suggestions, communicating with front-line operation and maintenance personnel to collect types of operation and maintenance faults, solutions and current outstanding problems, collecting on-site fault information and technical processing requirements and forming reports.

The standard of fault diagnosis is established. The business process, standard steps and diagnosis methods of fault treatment are studied. The fault classification diagram, processing flow chart and data flow chart are drawn up, and the fault diagnosis standard and treatment standard are compiled.

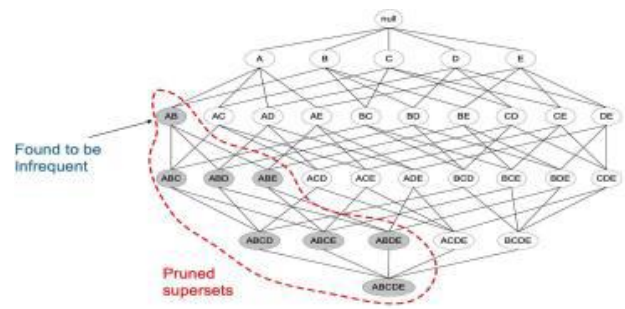

The fault diagnosis algorithm is studied. According to the actual needs of field operation and maintenance, the data sources for current fault, anomaly detection, early warning and location are studied, and the data format, particle, frequency and data characteristics are analyzed. By using advanced technologies such as data mining, rule engine and knowledge atlas, a series of arithmetic models that can accurately and timely diagnose faults are formed.

The acquisition fault diagnosis engine is established. From the aspects of business, application and technology, the overall architecture is designed, using large data, artificial intelligence and other technical means to design a multi-dimensional integrated accurate diagnosis engine, which can realize automatic fault monitoring and perception, intelligent diagnosis and intelligent repair, and solve the precise diagnosis of acquisition failures, qualitative analysis of equipment failures and guidance of the operation process of on-site fault treatment, thus forming a comprehensive accident. Barrier Intelligent Diagnosis System.

\subsection{Data Source and Model Construction}

\subsubsection{Off-line model.}

Based on the information of intelligent acquisition terminal reporting outage events, operator and SIM card business information, PMS planned outage record data, terminal file parameter information, software version and upgrade information, field operation and maintenance feedback and test results, the centralizer off-line outage diagnosis model is constructed, which realizes the analysis of off-line cause characteristics and remote fault repair of centralizer terminal, and intelligently patrols terminal faults. Check provides model support.

$$
\begin{aligned}
\operatorname{support}(A \Rightarrow B) & =P(A \cup B) \\
\operatorname{confidence}(A \Rightarrow B) & =P(B \mid A) \\
& =\frac{P(A \cup B)}{P(A)} \\
\operatorname{lift}(A \Rightarrow B) & =\frac{\operatorname{confidence}(A \Rightarrow B)}{P(B)} \\
& =\frac{P(A \cup B)}{P(A) P(B)}
\end{aligned}
$$

Figure 1. Data Factor Mining Diagram and Apriori Algorithms

Based on the acquired data, the support factors of concentrator off-line under the same line are judged. The Apriori algorithm is used to mine association rules with the extracted data factors, and the data model of association rules is produced.[3]

\subsubsection{Logical regression model.}

Based on file parameters, clock battery voltage, fault report and batch data, combined with downlink channel and equipment operation and maintenance results, a fault analysis and diagnosis model is constructed to realize concentrator stop-copying fault analysis and remote acquisition anomaly repair.

$$
\ln \frac{p}{1-p}=\beta_{1} x_{1}+\beta_{2} x_{2}+\beta_{3} x_{3}+\beta_{4} x_{4}+\beta_{5} x_{5}+\mu
$$

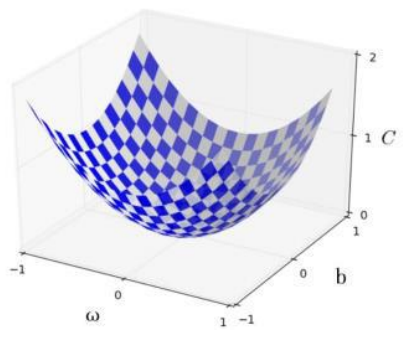

Figure 2. Logical regression model

The stopping faults of intelligent acquisition terminal are set as dependent variables, and the influence factors 
of stopping are set as independent variables. The coefficients of each independent variable are calculated. According to the gradient descent algorithm, the optimal weights are found to judge the main reasons of stopping the terminal and the importance of each influencing factor, and a logistic regression model is generated.[4]

\subsubsection{Missed copy model.}

Based on the detailed data of ammeter parameters, master station parameters, software version, ammeter clock and battery voltage data, carrier equipment and carrier interference source detection information, 485 working conditions, the fault analysis and diagnosis model of concentrator leak reading is constructed, which realizes leak reading reason analysis and remote fault repair, and provides model support for fault analysis of full data acquisition.

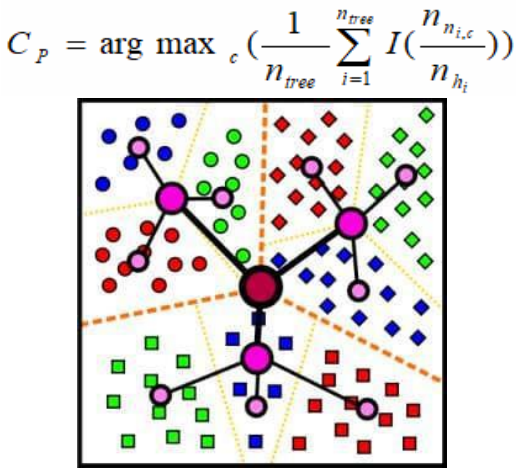

Figure 3. Decision Tree Nodes and Splitting Algorithms

The eigenvector is constructed to determine the decision result of a node in the decision tree. According to the nodes of each tree, the characteristics are selected randomly, the optimal splitting mode is calculated, and the above steps are repeated to construct another decision tree until a group of decision trees reach a predetermined number, that is, the random forest model is finally generated.[5]

\section{Follow-up Improvement of Fault Diagnosis Model for Intelligent Acquisition Terminal}

The basic purpose of fault diagnosis model is to guide grass-roots operation and maintenance personnel to narrow the scope of judgment and improve the efficiency of operation and maintenance, and the accuracy of diagnosis model is one of the key points for the follow-up improvement of the model. Through collecting the actual working environment of the operation and maintenance site, feedback from the pilot power supply companies, testing the model rules, optimizing and improving the existing model rules, and consolidating the rule base.

In the follow-up implementation and application process, we will continue to collect and sort out fault models, supplement existing rules, enrich fault rule base, further strengthen the research of typical and difficult fault diagnosis models concerned by grass-roots units, such as signal interference, software anomalies, interface faults, strengthen practical exploration, dig in-depth characteristics of various fault data, improve and enrich and accurately select model parameters.

The data in Table 2 are the data of pilot application of the model in Liaoning Province.

Table 2. Application of Fault Diagnosis Model for Intelligent Acquisition Terminal

\begin{tabular}{cccc}
\hline Month & $\begin{array}{c}\text { Acquisition of Abnormal } \\
\text { Number by Model Diagnosis }\end{array}$ & $\begin{array}{c}\text { On-site verification } \\
\text { confirms the number of } \\
\text { anomalies }\end{array}$ & Model accuracy \\
\hline 6 month & 154 & 123 & $79.87 \%$ \\
\hline 7 month & 126 & 105 & $83.33 \%$ \\
\hline 8 month & 113 & 98 & $86.73 \%$
\end{tabular}

\section{Concluding remarks}

Electricity information acquisition system is an important part of "three types, two networks" and the technical basis of intelligent electricity service. The safe and reliable operation of acquisition equipment is directly related to the development level of "informationization, automation and interaction" in the ubiquitous Internet of Things. In order to realize the "full coverage, full acquisition and full cost control" of power customers within the scope of the State Grid Corporation as soon as possible, the ability of intelligent diagnosis, analysis and processing of acquisition anomalies needs to be strengthened urgently. This paper makes a multi-angle analysis of the existing problems in fault diagnosis of intelligent acquisition terminal, comprehensively collects power information to collect on-site fault information, investigates the technical requirements of on-site fault treatment, uses large data analysis and artificial intelligence technology to collect on-site fault intelligent diagnosis model, and solves the precise diagnosis of acquisition fault, qualitative analysis of equipment fault and on-site fault treatment.[6]

\section{Reference}

1. Xin He, Jie Du, Lu Yin. (2019) Application of 
Electricity Information Acquisition System in Distribution Network Operation and Maintenance Management. Electronic Products World, (3):49-50.

2. Shuran Zhai, Jingya Lu. (2018) Exploration and practice of operation and maintenance system of electric power information acquisition system. Communication power technology,35(11): 169-170.

3. Liwei Zhou. (2017) Research on Markov Prediction Model of Power Transformer Fault Based on Association Rule Analysis. Chongqing: Chongqing University.

4. Jieyi Deng. (2018) Research and application of multi-classification logistic regression algorithm in large data environment. Wuhan: Wuhan University of Engineering.

5. Xianzheng Feng,Xiaojun Liao.(2019)Fault diagnosis method of virtual circuit in intelligent substation based on decision tree algorithm. Rural electrification, (8): 43-44.

6. Caixia Zhao, Yuzhu Zhang, Anjun Wang. (2017) Brief discussion on the influencing factors of the success rate of electricity information acquisition. Engineering Technology, (11):171. 